\title{
EURECCA colorectal: Multidisciplinary Mission statement on better care for patients with colon and rectal cancer in Europe
}

\author{
Cornelis J.H. van de Velde ${ }^{\mathrm{a}, *}$, Cynthia Aristei ${ }^{\mathrm{b}}$, Petra G. Boelens ${ }^{\mathrm{c}}$, \\ Regina G.H. Beets-Tan ${ }^{\mathrm{d}}$, Lennart Blomqvist ${ }^{\mathrm{e}}$, Josep M. Borras ${ }^{\mathrm{f}, \mathrm{g}}$, Colette B.M. \\ van den Broek ${ }^{c}$, Gina Brown ${ }^{\mathrm{h}}$, Jan-Willem Coebergh ${ }^{\mathrm{i}}$, Eric Van Cutsem ${ }^{\mathrm{j}}$, \\ Eloy Espin ${ }^{k}$, Jola Gore-Booth ${ }^{1}$, Bengt Glimelius ${ }^{\mathrm{m}}$, Karin Haustermans ${ }^{\mathrm{n}}$,
} Geoffrey Henning ${ }^{1}$, Lene H. Iversen ${ }^{\circ}$, J. Han van Krieken ${ }^{p}$, Corrie A.M. Marijnen ${ }^{q}$, Pawel Mroczkowski ${ }^{r}$, Iris Nagtegaal ${ }^{p}$, Peter Naredi ${ }^{\mathrm{s}}$, Hector Ortiz ${ }^{\mathrm{t}}$, Lars Påhlman ", Philip Quirkev ${ }^{\mathrm{v}}$, Claus Rödel ${ }^{\mathrm{w}}$, Arnaud Roth ${ }^{\mathrm{x}}$, Harm J.T. Rutten ${ }^{\mathrm{y}}$, Hans J. Schmoll ${ }^{\text {, Jason Smith }}{ }^{\text {aa }}$, Pieter J. Tanis ${ }^{\text {ab }}$, Claire Taylor ${ }^{\text {ac }}$, Arne Wibe ${ }^{\text {ad }}$, Maria Antonietta Gambacorta ae, Elisa Meldolesi ae, Theo Wiggers af, Andres Cervantes ${ }^{\text {ag }}$, Vincenzo Valentini ${ }^{\text {ah }}$

\footnotetext{
${ }^{a}$ Chairman EURECCA and CC3, Executive Board of ECCO, Department of Surgery, Leiden University Medical Center, The Netherlands

${ }^{\mathrm{b}}$ Executite Committee CC3, Radiation Oncology Section, Department of Surgery, Radiology and Dentistry, University of Perugia, Italy

'Scientific Committee CC3, Research Fellow EURECCA, Department of Surgery, Leiden University Medical Center, The Netherlands

${ }^{\mathrm{d}}$ European Saciety Radiology, Department of Radiology, Maastricht University Medical Center, Maastricht, The Netherlands

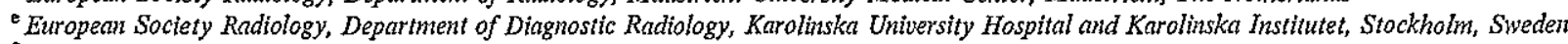

${ }^{f}$ ECCOIEPAAC Catalonian Cancer Strategy Unit, Department of Health, Catalonian Regional Authority, L'Hospitalet de Llobregat,

Barcelona, Spain

'Department of Clinical Sciences, Belluitge Biomedical Research Institute, University of Barcelona, Feixa Llarga S/N, L'Hospitalet de Llobregat, Barcelona, Spain

${ }^{\mathrm{h}}$ European Soctety Radiology, Department of Radiology, The Royal Marsden NHS Foundation Trust, Fulham Road, London, UK

${ }^{\dagger}$ Erasmus MC Rotterdam, and Research Department, Comprehensive Cancer Centre South, Eindhoven, The Netherlands

${ }^{j}$ Executive Bourd ESMO, Medical Director EuropaColon, Digestive Oncology Unit, University Hospital Gasthwisberg, Leuven, Belginan

${ }^{\mathrm{k}}$ Registry Delegate Colorectal Sirgery Unit, Hospital Valle de Hebroin, Autonomous University of Barcelona, Barcelona, Spain

${ }^{1}$ EUROPACOlon Representatives, CEOIFounter and Policy Director

${ }^{m}$ ESTRO Department of Radiology, Oncology and Radiation Sciences, Uppsala University, Uppsala, Sweden

${ }^{n}$ ESTRO, EORTC, Registry Delegate, Department of Radiation Oncology, University Hospitals Lewven Campus Gasthuisberg, Leuten, Belgitum

${ }^{\circ}$ Registry Delegate, Aarhus University Hospital, Department of Surgery, Aarhus, Denmark

${ }^{\mathrm{P}}$ ESP, Department of Pathology, Radboud University Nijmegent Medical Center, Nijmegen, The Netherlands

'ESTRO, Department of Radiation Oncology, Leiden University Medical Center, The Netherlands

'ESCP, Registry Delegate, Department of General, Visceral and Vascular Surgery, Otto-von-Guericke University of Magdeburg, Germany

' ESSO, Past-President, Department of Surgery, Institute of Clinical Sctences, Sahlgrenska Academy at University of Gothenburg, Sahlgrenska University Hospital, Gothenburg, Sweden

'Registry Delegate, Spanish Soclety of Surgeons, ESCP, Department of Surgery, Public University of Navarra, Spain

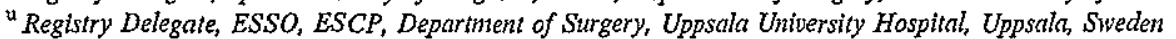

${ }^{\vee}$ ESP, Pathology and Tumour Biology, Leeds Institute of Molecular Medicine, St. James's University Hospital, Leeds, UK

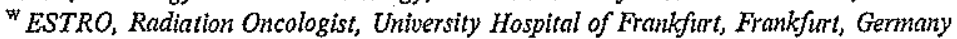

${ }^{\mathrm{x}}$ ESSO, Oncosurgery Unit, HUG, Geneva, Sivitzerland
} 


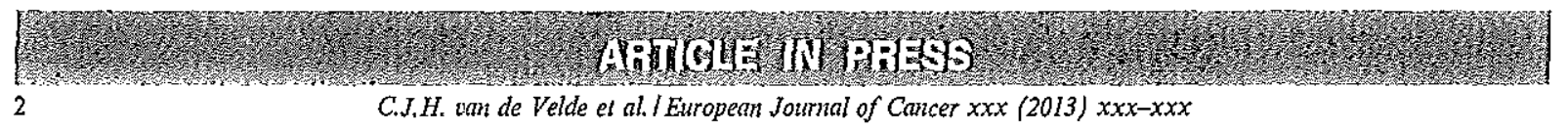

${ }^{y}$ ESSO, Department of Surgery, Catharina Hospital Eindhoven, Eindhoven, The Netherlands

${ }^{z}$ ESMO, Department of Oncology/Haematology, Martin Luther University Halle, Germany

${ }^{2 a}$ ESSO, Registry Delegate, Department of Colorectal Surgery, West Middlesex University Hospital, Isleworth, UK

${ }^{\mathrm{ab}}$ Representative Laparoscopic CR Surgery, ESSO, Department of Surgery, Academic Medical Center, Amsterdam, The Netherlands

${ }^{20}$ EONS Representative, Lecturer and Macintlan Lead Colorectal CNS, St. Mark's Hospital, Harrow, Middlesex, UK

${ }^{a d}$ ESSO, ESCP, Registry Delegate, Department of Surgery, St. Olavs Hospital, Trondheim University Haspital, Trondheim, Norway

${ }^{2 a}$ Scientific Committee, Cattedra di Radioterapta, Università Cattolica S. Cuore, Rome, Italy

af ESSO, Registry Delegate, Department of Surgical Oncology, University Medical Center Groningen, University of Groningen, Groningen, The Netherlands

${ }^{2 z}$ Executive Board ESMO, Institute of Health Research INCLIVA, University of Valencia, Spain

${ }^{\text {ah }}$ Executive ommittee CC3, ESTRO, Professor of Radiation Oncology, Cattedra di Radioterapia, Universitì Cattolica S. Cuore, Rome, Italy

\section{KEYWORDS}

Quality assurance

Multidisciplinary team

Consensus

Delphi method

Audit

Colon cancer

Rectal cancer

Neoadjuvant radiother-

apy

Neoadjuvant chemora-

diotherapy

Minimal invasive surgery

\begin{abstract}
Background: Care for patients with colon and rectal cancer has improved in the last twenty years however still considerable variation exists in cancer management and outcome between European countries. Therefore, EURECCA, which is the acronym of European Registration of cancer care, is aiming at defining core treatment strategies and developing a European audit structure in order to improve the quality of care for all patients with colon and rectal cancer. In December 2012 the first multidisciplinary consensus conference about colon and rectum was held looking for multidisciplinary consensus. The expert panel consisted of representatives of European scientific organisations involved in cancer care of patients with colon and rectal cancer and representatives of national colorectal registries.
\end{abstract}

Methods: The expert panel had delegates of the European Society of Surgical Oncology (ESSO), European Society for Radiotherapy \& Oncology (ESTRO), European Society of Pathology (ESP), European Society for Medical Oncology (ESMO), European Society of Radiology (ESR), European Society of Coloproctology (ESCP), European CanCer Organisation (ECCO), European Oncology Nursing Society (EONS) and the European Colorectal Cancer Patient Organisation (EuropaColon), as well as delegates from national registries or audits. Experts commented and voted on the two web-based online voting rounds before the meeting (between 4th and 25th October and between the 20th November and 3rd December 2012) as well as one online round after the meeting (4th-20th March 2013) and were invited to lecture on the subjects during the meeting (13th-15th December 2012). The sentences in the consensus document were available during the meeting and a televoting round during the conference by all participants was performed. All sentences that were voted on are available on the EURECCA website www.canceraudit.et.

The consensus document was divided in sections describing evidence based algorithms of diagnostics, pathology, surgery, medical oncology, radiotherapy, and follow-up where applicable for treatment of colon cancer, rectal cancer and stage IV separately. Consensus was achieved using the Delphi method.

Results: The total number of the voted sentences was 465 . All chapters were voted on by at least $75 \%$ of the experts. Of the 465 sentences, $84 \%$ achieved large conșensus, $6 \%$ achieved moderate consensus, and $7 \%$ resulted in minimum consensus. Only $3 \%$ was disagreed by more than $50 \%$ of the members.

Conclusions: It is feasible to achieve European Consensus on key diagnostic and treatment issues using the Delphi method. This consensus embodies the expertise of professionals from all disciplines involved in the care for patients with colon and rectal cancer. Diagnostic and treatment algorithms were developed to implement the current evidence and to define core treatment guidance for multidisciplinary team management of colon and rectal cancer throughout Europe.

(c) 2013 Elsevier Ltd. All rights reserved.

\footnotetext{
* Corresponding author; Address: Leiden University Medical Center, Department of Surgery, K6-R, P.O. Box 9600, 2300 RC Leiden, The Netherlands. Tel.: +3171526 2309; fax: +31715266750 .

E-mail address: c.j.h.van_de_velde@lumc.nl (C.J.H. van de Velde).
} 


\section{Introduction}

Colon and rectal cancer (CRC) are the second most common cancers $(1,234,000$ cases worldwide in 2008 according to GLOBOCAN and 342,137 in 27 country's in Europe in 2012) and cause many cancer related deaths each year (149,984 cases in Europe in 2012). ${ }^{1,2}$ The first two multidisciplinary consensus meetings on key issues in rectal cancer were held in 2004 and 2008 in Perugia, Italy. Because of the observed variation in incidence, treatment and outcome of colon and rectal cancer worldwide, the Third European Consensus meeting in December 2012 was organised for colon and rectal cancer. The meeting aimed to outline the 'core quality treatment strategies' for colon and rectal cancer and reach consensus using the Delphi Method as applied in the previous editions. ${ }^{3}$ In short, we invited a multidisciplinary expert panel consisting of representatives of European scientific organisations involved in providing cancer care to colon and rectal cancer patients, in order to secure a firm basis to reach the health professionals in the field.

The mission of the European CanCer Organisation (ECCO) aims at 'Every patient deserves the best treatment there is'. To optimise cancer care for patients with colon and rectal cancer, one of the key challenges is to strive for optimal multidisciplinary management of outcome besides reaching a European consensus. High incidence and potentially high curability of colon and rectal cancer accentuate that these patients deserve full attention and effort of a multidisciplinary team both before neoadjuvant treatment or primary surgery as well as after surgery to decide on treatment strategies.

The EUROCARE project, a European Union project to assemble survival data from population-based cancer registries, showed wide variation in rectal and colon cancer 5-year cumulative survival between different European countries in the nineties. ${ }^{4-6}$ Due to nonacceptable results, several countries started quality registries and subsequently quality programmes were initiated based on these reports. The different features of health care in Europe were explored and revealed that there is still a wide diversity of national guidelines and routine clinical practice and that every country has a different health care system, infrastructure and a different availability of registration of population based data. ${ }^{7,8}$

Since the beginning of the 1990 s treatment of colorectal cancer has changed substantially. At present, many countries have access to national and international guidelines. ' Adherence to guidelines is not always explored or monitored; improvements in securing patterns of care are still ahead. Ideally, treatment decisions are nowadays made preoperatively and postoperatively in multidisciplinary boards. While later reports of EUROCARE showed that although survival was improving, inter-country variation is still persisting, suggesting room for further improvement. ${ }^{5,10}$ Even in high-income countries with well established guidelines and a similar healthcare structure, the difference in outcome is unexplained and vast. ${ }^{1}$ Highly relevant changes in the therapeutic approach have taken place in recent years such as the implementation of the total mesorectal excision (TME)-technique for rectal cancer surgery. ${ }^{14}$ Another example of progress is preoperative treatment including radiotherapy and chemo radiotherapy for patients with rectal cancer and the incorporation of adjuvant chemotherapy for patients with colon cancer. ${ }^{12-17}$ In the field of diagnostic imaging, primary staging has been improved, by introducing magnetic resonance imaging (MRI) in the preoperative work-up for rectal cancer ${ }^{18,19}$ and optimised computed tomography (CT) also contributed to more accurate staging. Structured examination of surgical specimen, such as number of lymph nodes and circumferential resection margin (CRM), leads to better postoperative identification of high risk patients. ${ }^{20}$ More and more countries are implementing screening programmes for $\mathrm{CRC}$, and guidelines for a high quality colorectal cancer screening in Europe have been published. ${ }^{21} \mathrm{~A}$ meta-analysis of randomised controlled trials reported that screening using flexible endoscopy reduces the incidence and mortality of colorectal patients. ${ }^{22}$ Furthermore, treatment of patients with stage IV became more successful with broader acceptance of liver resection and improved chemotherapy regimens. Overall, survival. has improved in most European countries over the past 20 years. In 19881990 survival of patients with rectal cancer was lower than that of patients with colon cancer. Survival of rectal cancer nowadays surpasses the survival of colon cancer (in North Europe, United Kingdom [UK] and central Europe). ${ }^{23} \mathrm{Clinical}$ audits were set up and several international trials were performed to improve loco regional control and survival of rectal cancer patients. ${ }^{24-29}$

Based on the benefits achieved by national audits, European Society of Surgical Oncology (ESSO) has initiated the EURECCA-project in partnership with European Society for Radiotherapy \& Oncology (ESTRO), European Society for Medical Oncology (ESMO), European Society of Coloproctology (ESCP), ECCO, and European Organisation for Research and Treatment of Cancer (EORTC). EURECCA is the acronym of European Registration of Cancer Care which aims to improve cancer outcome in Europe by comparing treatment strategies and outcome of national audits. ${ }^{30}$ In order to update the European consensus of multidisciplinary treatment guidelines, the Third European Consensus Conference Colon \& Rectum was held in Perugia, Italy from 13th till 15th December 2012.

\section{Methodology}

Consensus was achieved by the Delphi Method using online web-based voting by experts and televoting 
Table 1

Examples of sentences voted during the Consensus in Colon and Rectum Cancer Care

Obtain colonoscopy \& biopsy preoperatively if possible. Completing colonoscopy to be performed soon after surgery if incomplete

MRI is mandatory in staging of all rectal cancers. Always describe cTNM and MRF, LN morphology in MRI report. Describe EMVI

Lesser choice exams for location are sigmoidoscopy (only distal), Double Barium Contrast Enema, CT-abdomen

CT-colonography could be considered only if necessary after an abdominal $\mathrm{CT}$

Abdominal and chest CT for distant metastases is recommended

Consider MRI liver for additional imaging of metastases if necessary

There is no role for PET/CT scan in primary staging of colon cancer

Bone or Brain imaging is recommended if symptoms are present

Abdominal and clest CT for distant metastases is recommended

Consider MRI liver for additional imaging of metastases if necessary

There is no role for PET/CT scan in primary staging of colon cancer

Bone or Brain imaging is recommended if syrnptoms are present

Pathology

Describe the used version of TNM and TNM stage in Pathology report

Describe all margins, complete resection and perforation if applicable

Describe lymph node number and number of positive nodes

Describe other possible predictors of poor outcome; less than $10 \mathrm{LN}, \mathrm{T} 4$ tumours,

lymphovascular invasion, extent of tumour spread beyond the musculairs

propria, poor differentiation

Surgery

R0 polypectomy of Tis or $\mathrm{Tl} \mathrm{sml}$, without lymphovascular invasion and no poor differentiation invasion could be considered for follow up

Fast track protocols when possible

Anatomical resection following the embryological planes is essential

Training according to EAES guidelines, relative contraindications are obesity, previous open abdominal surgery and locally advanced disease

Laparoscopic colectomy enhances postoperative recovery and has similar outcomes (survival) to open surgery in selected patients. Attention late/reactive converted patients do worse than open

Consider Stenting as a bridge to surgery, be aware of risks of perforation, occlusion

Describe the used version of TNM and TNM stage in Pathology report

Describe all margins, complete (mesorectum in TI-3) resection and perforation if applicable Always describe CRM in mm from tumour free maxgin

Describe lymph node number and number of positive nodes

Describe other possible predictors of poor ontcome; T4 tumours, lymphovascular invasion, extent of tumour spread beyond the musculairs propria, poor differentiation

R0 polypectomy of Tis or T1 smi, without lymphovascular invasion and no poor differentiation invasion could be considered for follow up

If local excision is considered TEM is the procedure to perform

Anatonical resection on careful preoperative planning based on MRI. TME surgery if possible is the gold standard

Respect leaming curve and EAES guidelines for laparoscopic TME surgery

Chemotherapy

No role for neoadjuvant chemotberapy in stage I-III

Chemotherapy in stage $X$ is not recommended

Adjuvant chemotherapy in stage II high risk could be considered

Chemotixerapy in stage $I$ is not recommended

Adjuvant chemotherapy in rectal cancer is to be considered in pathological stage $\mathrm{II} / \mathrm{WI}$ 
Table 1

Examples of sentences voted during the Consensus in Colon and Rectum Cancer Care.

\section{Colon cancer \\ Adjuvant chemotherapy in stage III and postoperative chemotherapy in stage IV is} recommended

\section{Radiotherapy}

Only consider $\mathrm{RT}$ in selected $\mathrm{T} 4$ colon cancer patients with residual disease

Follow up

More research needed

CEA

Colonoscopy

In high risk patients consider annual CT

CT or PET/CT only in patients with positive findings on routine follow up imaging

Consider at least 5 year follow up

Abbreviations: CT, computed tomography; MRI, magnetic resonance imaging; PET, positron emission tomography; TNM, classification of malignant tumours; LN, lymph node; R0, no residual tumour; T4 Tumour. invasion of other organs; Tis Tumour, carcinoma in situ; sml, classification by Kudo; When less than one-third of the submucosa is invaded the stage is sml, and if more than twothirds is invaded the stage is sm3, while stage sm2 is intermediate with invasion of cancer into the middle third. $\mathrm{Sm} 1$ is when the depth of invasion is less $\leqslant 1$ mm or $1000 \mu \mathrm{m}$ from the muscularis mucosae. EAES, European Association for Endoscopic Surgery; ERAS, enhanced recovery after surgery; CEA, carcinoembryonic antigen; MRF, meso rectal fascia; CRM, circumferential resection margin; RT, radiation therapy, Gy, gray; RCT, chemoradiation; TME, total mesorectal excision.
Rectal cancer

Adjurvant chemoradiotherapy could be considered if no preoperative radiotherapy was given. that preoperative radiotherapy is better

Adjwant chemotherapy can be considered after any preoperative treatment in stage $\mathrm{I} / \mathrm{III}$

No neoadjuvant treatment is recommended in early stages (cT1-2 N0 MO)

For high rectal tumours $\mathrm{T} 3 \mathrm{a} / \mathrm{b}$ no preoperative $\mathrm{RT}$ is recommended

cT3 (MRRF-) NO MO consider three treatments;

1. TME surgery and observation,

2. $5 \times 5$ Gy and immediate TME surgery,

3. chemoradiation followed by delayed TME surgery

c13 c/d (MRF-) or N + MO recommend chenzoradiotherapy before TME surgery

cT3 (MRF+) any N, M0 or cT4, any N, M0 preoperative downstaging with chemoradiotherapy,

followed by TME surgery or extramesorectal excision (exenteration)

\section{More research needed}

CEA

Colonoscopy

tients consider annual CT 
during the meeting. The multidisciplinary expert panel consisted of representatives of European scientific organisations involved in cancer care of patients with colon and rectal cancer and representatives of national colorectal registries. The following organisations were involved; European Society of Surgical Oncology (ESSO), European Society for Radiotherapy \& Oncology (ESTRO), European Society of Pathology (ESP), European Society for Medical Oncology (ESMO), European Society of Radiology (ESR), European Society of Coloproctology (ESCP), European CanCer Organisation (ECCO), European Oncology Nursing Society (EONS) and the European Colorectal Cancer Patient Organisation (EuropaColon). Experts commented and voted on the two online voting rounds before the meeting (4th-25th October 2012 and 20th November until the 3rd December 2012) as well as one online round after the meeting (4th-20th March 2013) and were invited to lecture on the subjects during the meeting (13th-15th December 2012). The sentences in the consensus document were available during the meeting and a televoting round during the conference by all participants was performed. All sentences that were voted on are available on the EURECCA website www.canceraudit.eu.

The consensus document was divided in sections describing evidence based algorithms of diagnostics, pathology, surgery, medical oncology, radiotherapy, and follow-up where applicable for treatment of colon cancer, rectal cancer and on stage IV separately.

\section{Results}

The Third Consensus Conference on Colon and Rectum, Perugia, developed the following mission statements;

\subsection{On audits and research}

National registries and audits are important to improve colorectal cancer survival. Definitions and guidelines should be comparable across Europe. Combining large national datasets can identify 'best practices'. Both randomised controlled trials and observational studies of large registries (national or European) are needed to identify key factors for the best colon and rectal cancer care. The strengths of large observational studies are related to providing outcome data on subgroups that are generally not included in clinical trials such as patients with co-morbidities and elderly. This will help professionals to optimise treatment strategies for these specific subgroups.

\subsection{On treatment}

Precision diagnosis will enable us to optimise staging and to individualise treatment. The mission is that every patient deserves the best. We need to continually review what is the best treatment, identify over and under-treatment, and determine the best care. We know that by working in a multidisciplinary environment together with specialist nurses and the patient, progress can be made. Examples of quality care treatment approaches discussed during the meeting are summarised in Table 1.

\subsection{On quality of care}

Given the importance of each entity within the colorectal cancer care process in determining outcome (surgery, pathology, diagnostic imaging (in staging and restaging), radiotherapy and chemotherapy), quality assurance programmes including education and training programmes should become mandatory for colon and rectal cancer services to provide the best quality of care. There is a need for accessible and transparent structures for cancer care in Europe.

Evidence based multidisciplinary management guidelines should be defined at national and European levels with the consensus of healthcare professionals, patient organisations and policy makers.

\section{Concluding remarks}

The Third Consensus Conference on colon and rectum held in December 2012 achieved large consensus in $84 \%$ of the sentences proposed, meaning that more than $95 \%$ of the experts agreed on these sentences. Reaching consensus is deemed feasible and achievable in a large number of key items related to diagnosis, staging and treatment using the Delphi method. The challenge remains to assess whether this new consensus reaches the field and will be practiced by physicians across Europe, because still large variations exist in clinical practice across Europe. EURECCA is a platform to assess clinical practice and quality, and to explore the relationship with survival. Also, different scientific societies and stakeholders could work together in order to build a EU consensus in one of the most frequent cancers diagnosed in European countries.

\section{Conflict of interest statement}

On behalf of the authors we state that the consensus meeting was possible due to a non-restrictive unconditional Grant from ESSO. No other conflict of interest for this publication.

\section{References}

1. Ferlay J, Shin HR, Bray F, Forman D, Mathers C, Parkin DM. Estimates of worldwide burden of cancer in 2003: GLOBOCAN 2008. Int J Comcer 2010;127(12):2893-917.

2. Ferlay J, Steliaroya-Foucher B, Lortet-Tieulent J, et al. Cancer incidence and mortality patterns in Europe: estimates for 40 countries in 2012. Eur $J$ Cancer 2013;49(6):1374-403. 


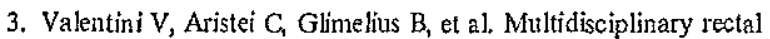
cancer munagement: 2nd European Rectal Cancer Consensus Conference (EURECA-CC2). Radiother Oncol 2009;92(2): 148-63.

4. Berrino F, De AR, Sant M, et al. Survival for eight major cancers and all cancers combined for European adults diagnosed in 1995-99: results of the EUROCARE-4 study. Lancet Oncol 2007;8(9):773-83.

5. Gatta $G$, Capocaccia R, Sant $M$, et al. Understanding yariations in survival for colorectal cancer in Europe: a EUROCARE high resolution study. Gut 2000;47(4):533-8.

6. Sant $M$, Capocaccia $R$, Verdecclia $A$, et al. Comparisons of colon-cancer survival among European countries: the Eurocare study. Int $J$ Cancer 1995;63(1):43-8.

7. Micheli A, Coebergh JW, Mugno E, et al. European health systems and cancer care. Ann Oncol 2003;14(Suppl 5):v41-60.

8. Siesling $S$, Kwast A, Gavin A, Baili $P$, Otter R. Availability of stage at diagnosis, cencer treatment delay and compliance with cancer guidelines as cancer registry indicators for cancer care in Europe: results of EUROCHIP-3 survey, Int J Cancer 2013:132:2910-7.

9. Schmoll HJ, Van CB, Stein A, et al. ESMO Consensus Guidelines for management of patients with colon and rectal cancer. A personalized approach to clinical decision making. Ann Oncol 2012;23(10):2479-516.

10. Morris EJ, Sandin F, Lambert $\mathrm{PC}$, et al. A population-based comparison of the survival of patients with colorectal cancer in England, Norway and Sweden between 1996 and 2004. Gut $2011 ; 6018): 1087-93$.

11. Maringe $C$, Walters $S$, Rachet $B$, et al, Stage at diagnosis and colorectal cancer survival in six high-income countries: a population-based study of patients diagnosed during 2000-2007. Acta Oncol 2013;52:919-32.

12. Ceelen WP, Van Nieuwenhove $Y$. Fierens $K$. Preoperative chemoradiation versis radiation alone for stage II and III resectable rectal cancer, Cochrome Datubise Syst Rev 2009:CD006041.

13. Des GG, Uzzan B, Morere JF, Perret G, Nicolas P. Duration of adjuvant chemotherapy for patients with non-metastatic colorectal cancer. Cochrane Database Syst Rev 2010(1):CD007046.

14. Kapiteijn E, Marijnen CA, Nagtegaal ID, et al. Preoperative radiotherapy combined with total mesorectal excision for resectable rectal cancer. $N$ Engl J Med 2001;345(9):638-46.

15. McCarthy K, Pearson K. Fulton R, Hewitt J. Pre-operative chemoradiation for non-metastatic locally advanced rectal cancer. Cachrome Database Syst Rev 2012;12:CD008368.

16. Sebag-Montefiore D, Stephens RJ, Steele R, et al. Preoperative radiotherapy versus selective postoperative chemoradiotherapy in patients with rectal cancer (MRC CR07 and NCIC-CTG COI0): a multicentre, randomised trial. Lancet 2009;373(9666):811-20.
17. van Gijn GW, Marijnen CA, Nagtegaal ID, et al. Preoperative radiotlierapy conbined with total mesorectal excision for resectable rectal cancer: 12-year follow-up of the multicentre, randomised controlled TME trial. Lancet Oncol 2011;12(6):575-82.

18. Mercury Study Group, Diagnostic accuracy of preoperative magnetic resonance imaging in predicting curative resection of rectal cancer: prospective observational study, BMJ 2006,333(7572), 779 .

19. Beets-Tan $R G_{r}$ Beets $G L$, Vliegen $R F$, et al. Accuracy of magnetic resonance imaging in prediction of tumour-free resection margin in rectal cancer surgery, Loncet 2001;357(9255):497-504.

20. Nagtegaal ID, Quirke P. What is the role for the circumferential margin in the modern treatment of rectal cancer? $J$ Clin Oncol 2008;26(2):303-12.

21. von KL, Patnick J, Segnan N, et al. Europenn guidelines for quality assurance in colorectal cancer screening and diagnosis: overview and introduction to the full supplement publication. Endoscopy 2013:45(1):51-9.

22. Elmunzer BJ, Hayward RA, Schoenfeld PS, Saini SD, Deshpande A, Waljee AK. Effect of flexible sigmoidoscopy-based screening on incidence and mortality of colorectal cancer: a systematic review and meta-analysis of randomized controlled trials. PLOS Med 2012;9(12):e1001352.

23. Brenner $H$, Bouvier AM, Foschi $R$, et al, Progress in colorectal caucer survival in Europe from the late 1980s to the early 21st century: the EUROCARE study. Im $J$ Cancer 2012;131(7):1649-58,

24. Bemstein TE, Endreseth BH, Romundstad P, Wibe A. Improved local control of reclal cancer reduces distant metastases. Colorectal Dis 2012;14(10):e668-78.

25. Iversen LH. Aspects of survival from colorectal cancer in Denmark, Don Med J 2012;59(4):B4428.

26. Ortiz $\mathrm{H}$, Wibe A, Ciga MA, et al. Impact of a multidisciplinary team training progranme on rectal cancer outcomes in Spain. Colorectal Dis 2013;15:544-51.

27. Pahlman $L$, Bohe $M$, Cedermark B, et al. The Swedish rectal cancer registry. $B r J$ Surg 2007;94(10):1285-92.

28. Penninckx $F$, Van EL, Michliels $G$, et al. Survival of rectal cancer patients in Belgium 1997-98 and the potential benefit of a national project. Acia Chir Belg 2006;106(2):149-57.

29. Wibe A, Moller B, Norstein J, et al. A national strategic change in treatment policy for rectal cancer-implementation of tofal mesorectal excision as routine treatment in Norway. A rational audit. Dis Colon Rectum 2002;45(7):857-66.

30. van Gijn GW, van de Velde C.. Quality assurance through outcome registration in colorectal cancer: an ECCO initiative for Europe. Acta Chir Itgos/ 2010;57(3):17-21. 